\title{
THE CONSTRUCTION AND DOCUMENTATION OF THE CELESCOPE CATALOG
}

\author{
K. HARAMUNDANIS
}

Smithsonian Institution, Astrophysical Observatory, Cambridge, Mass. 02138, U.S.A.

\begin{abstract}
In this paper, I describe briefly the instrument and the reduction system we have used for obtaining ultraviolet magnitudes and present some information concerning the sky areas and types of stars we have observed. I shall also comment on our use of catalogs in machine-accessible form and make suggestions concerning the most useful identifications to be assigned to observed objects, particularly those in the southern hemisphere.
\end{abstract}

\section{The Instrument}

In 1968, the Orbiting Astronomical Observatory OAO 2 was launched. Figure 1 is a picture of the satellite taken shortly before launch to give an idea of sits ize. The four Celescope photometers of the Smithsonian Astrophysical Observatory (SAO) were placed in one end of the satellite; the University of Wisconsin's experiment was in the other. In the photograph, the large solar paddles are fully extended and the upper sunshade is open.

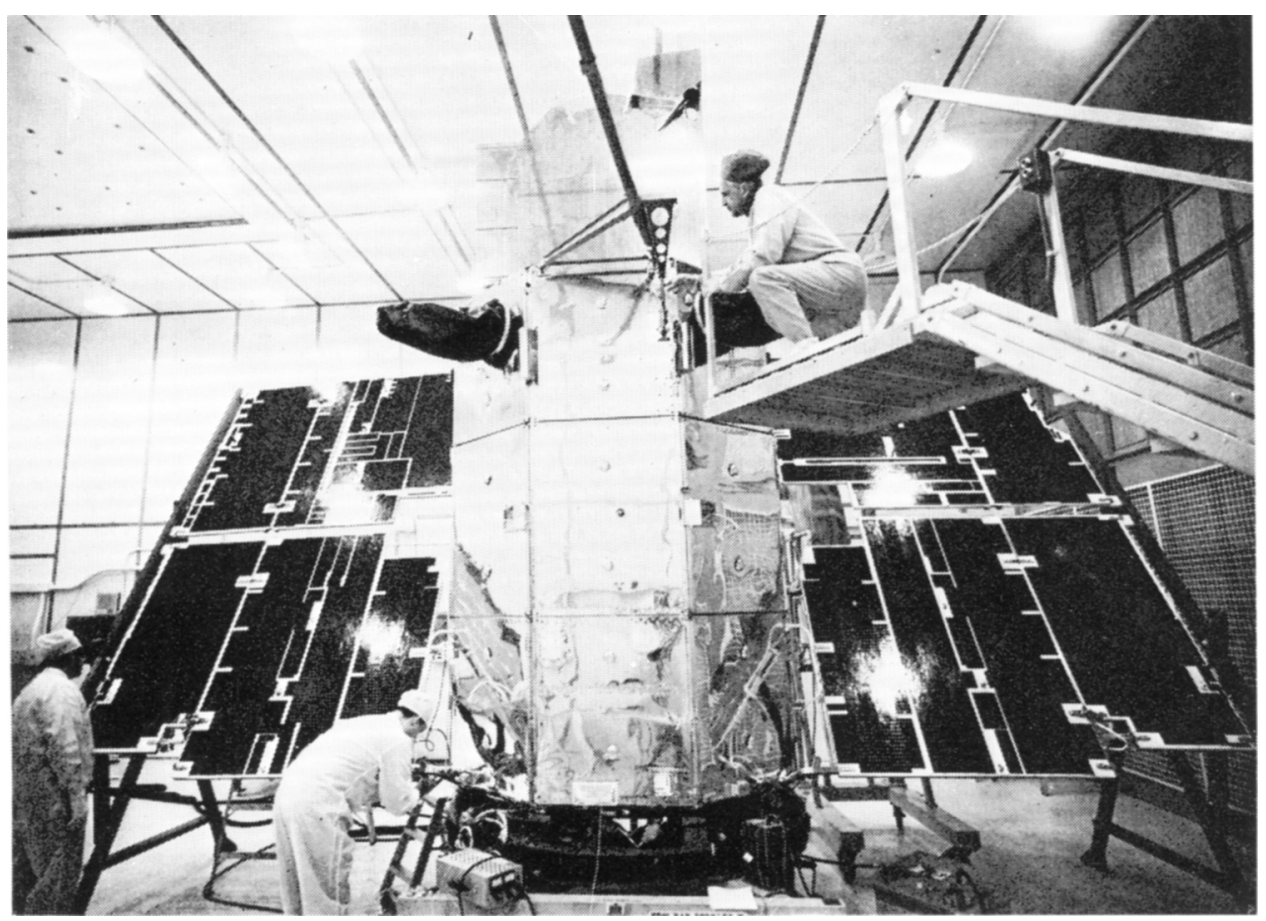

Fig. 1. OAO 2 during checkout procedures shortly before launch (December 1968) (courtesy of NASA). 


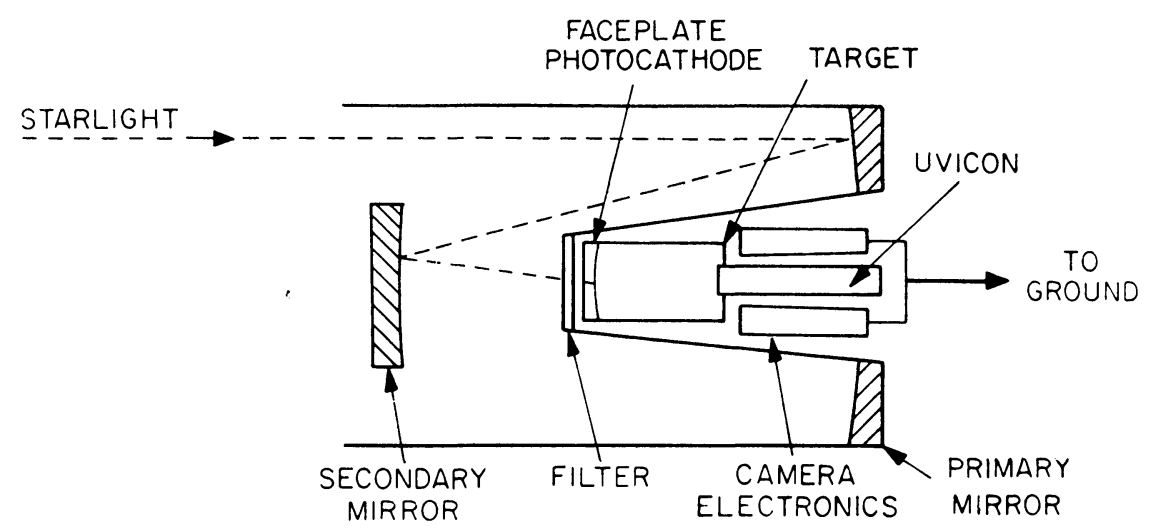

Fig. 2. Schematic diagram of Celescope photometer and telescope (from Nozawa, 1969).

A schematic diagram of one of the photometers is shown in Figure 2. The primary mirror is 12 in., and the filter is split into two sections in each of the four photometers so as to continue to provide data in more than a single broad passband in case of failure of any one of the cameras. The heart of the system is the Uvicon (photocathode,

TABLE I

Effective wavelength and halfwidth of Celescope filters

\begin{tabular}{lll}
\hline Filter & $\lambda_{\text {eff }}$ & $\Delta \lambda$ \\
\hline & & \\
U1 & 2582 & 550 \\
U2 & 2308 & 850 \\
U3 & 1621 & 325 \\
U4 & 1537 & 450
\end{tabular}

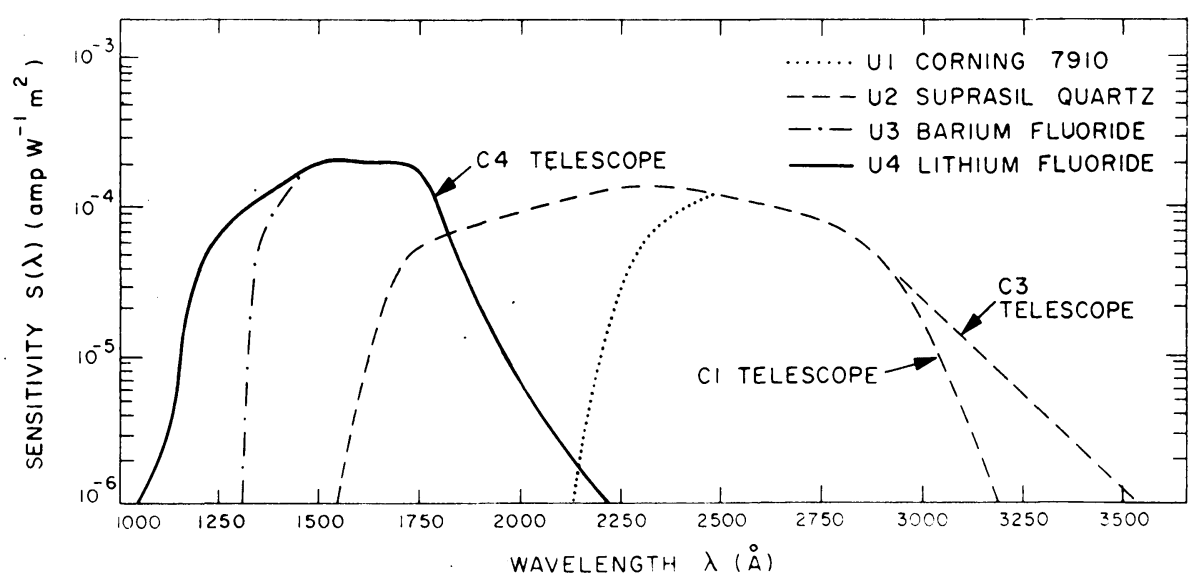

Fig. 3. Spectral response curves of Celescope (from Davis, 1968). 


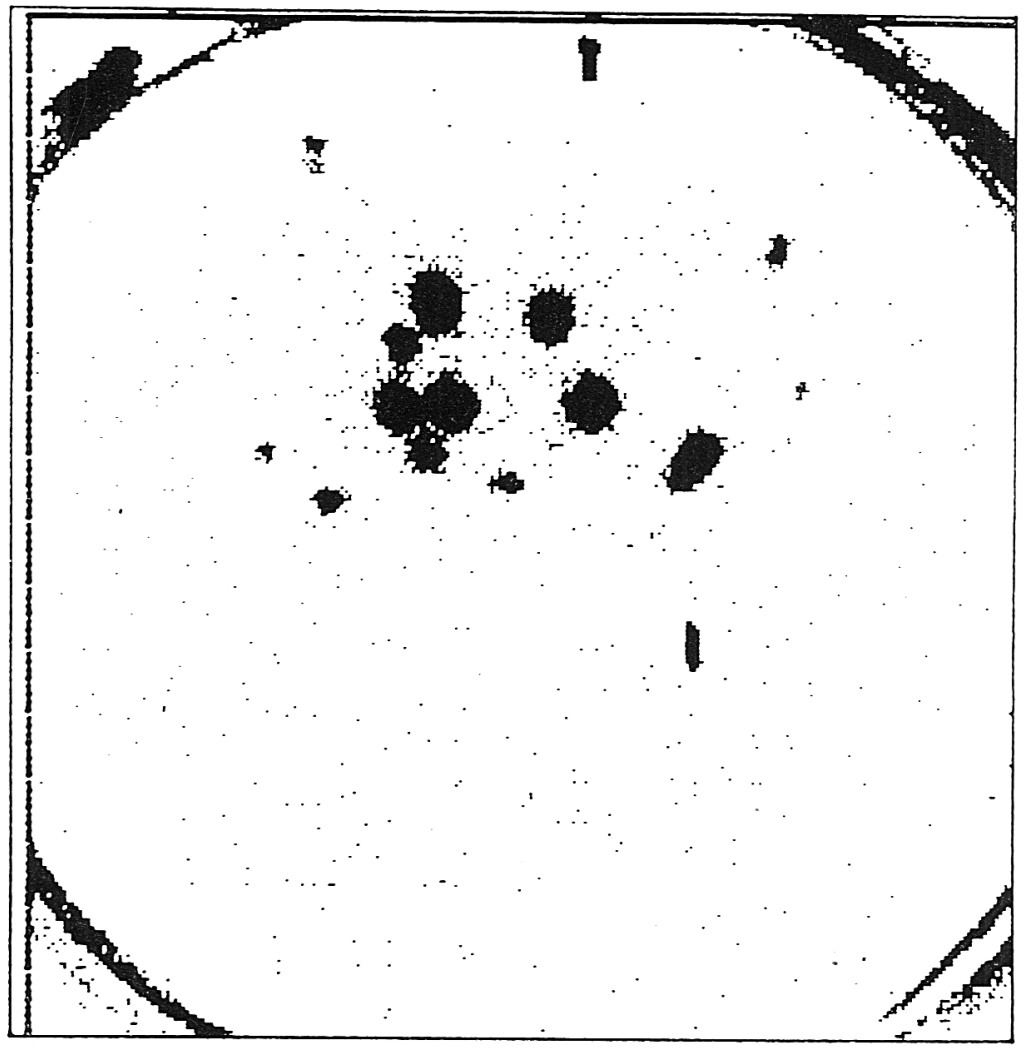

Fig. 4a. The Pleiades taken by Celescope.

target, and high-voltage power supply) described in numerous papers (cf. Nozawa, 1969; Celescope Staff, 1971).

With the four photometers, we observed stars over four passbands, two in each photometer for redundancy. The effective wavelength and half-width of each filter are given in Table I. The sensitivity of camera and filter combined is indicated in Figure 3. Note the difference between the U1 filters in cameras 1 and 3 (labeled C1 and C3). Figure 4 gives a single example of a Celescope picture. I have selected this picture of the Pleiades because it was taken midway in the lifetime of the experiment and was an exposure of only $5 \mathrm{~s}$. For comparison, a ground-based photograph of the cluster is also shown. All the stars visible in the Celescope picture are in the U4 filter, and all are of B spectral class. The faintest object in the picture is a $6.16 \mathrm{mag}$. B9V star just above the pair Atlas and Pleione. Recall that this is a $5 \mathrm{~s}$ exposure; our usual exposure time is $60 \mathrm{~s}$, which in this wavelength unfortunately is swamped by radiation from the geocorona. This swamping initially proved a major obstacle in our processing of the data but was effectively overcome by some program refinements; we have as yet, however, been unable to reduce adequately the magnitudes of stars observed in such a swamped picture. 


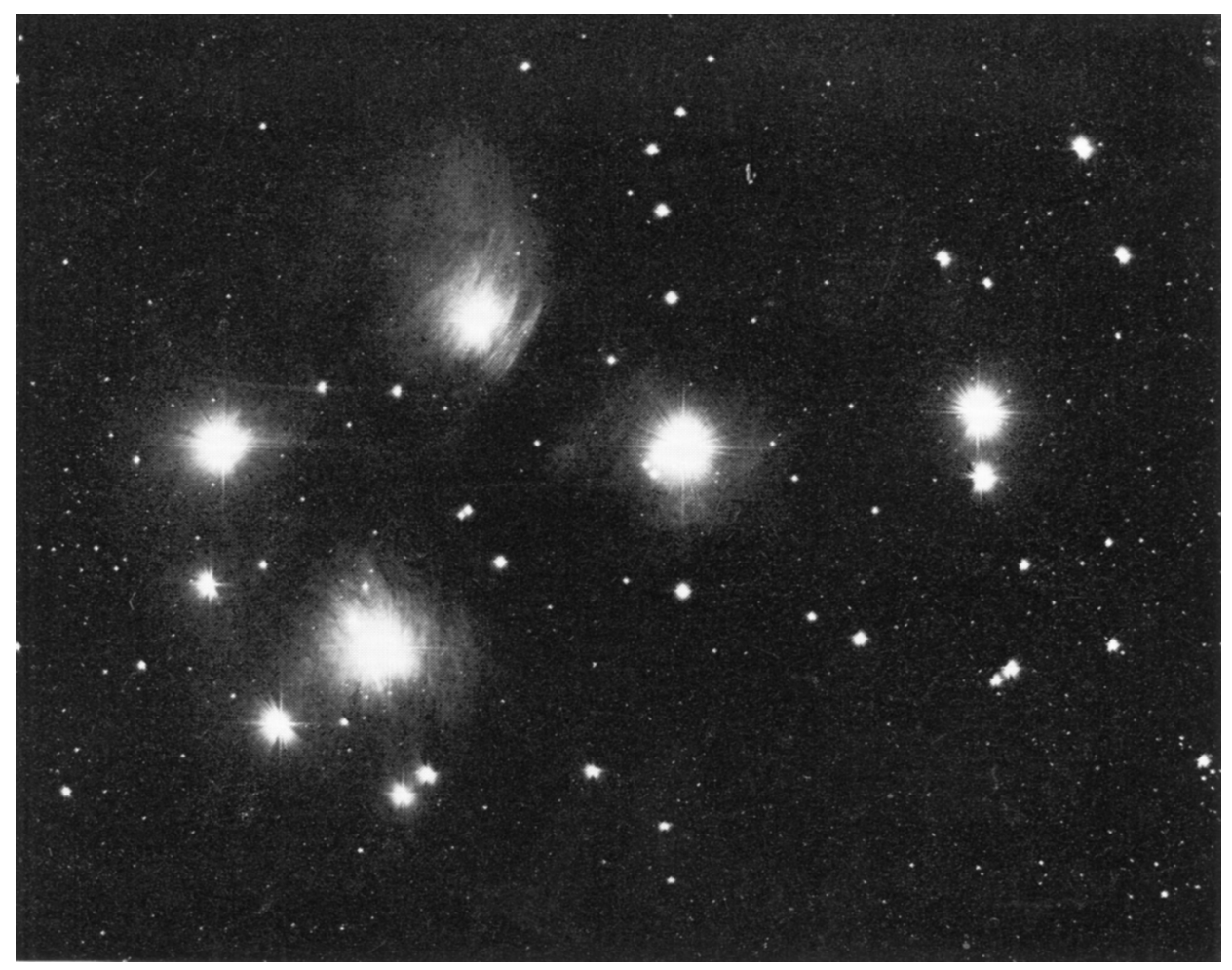

Fig. 4b. The Pleiades taken by the Crossley reflector (Lick Observatory).

\section{The Reductions}

Figure 5 is a diagram of the system we have developed for reducing the observations to ultraviolet magnitudes and for obtaining Durchmusterung identifications. The data, originally transmitted from the satellite to various ground stations are sent to Goddard Space Flight Center, where they are digitized. From there they are brought to SAO for our processing. It may be of interest that our processing at SAO is done on a CDC 6400 computer (maximum user capacity $140 \mathrm{~K}$ ). Our computer programs are largely written in FORTRAN, with some special routines in the CDC machine language COMPASS.

After verification of auxiliary information relating to pointing, temperatures, exposure time, and cameras, the data are sent out for conversion to picture displays. Some resolution is thereby lost, since the original data are quantized in 127 intensity levels while the displays comprise only 16 steps.

The digitized data in our main reduction system are passed into our signal-processing program, which treats each picture separately to determine background intensity and locate all non-noise objects. The significance level is allowed to differ for each picture and for different locations in the picture. The digitized objects are displayed 

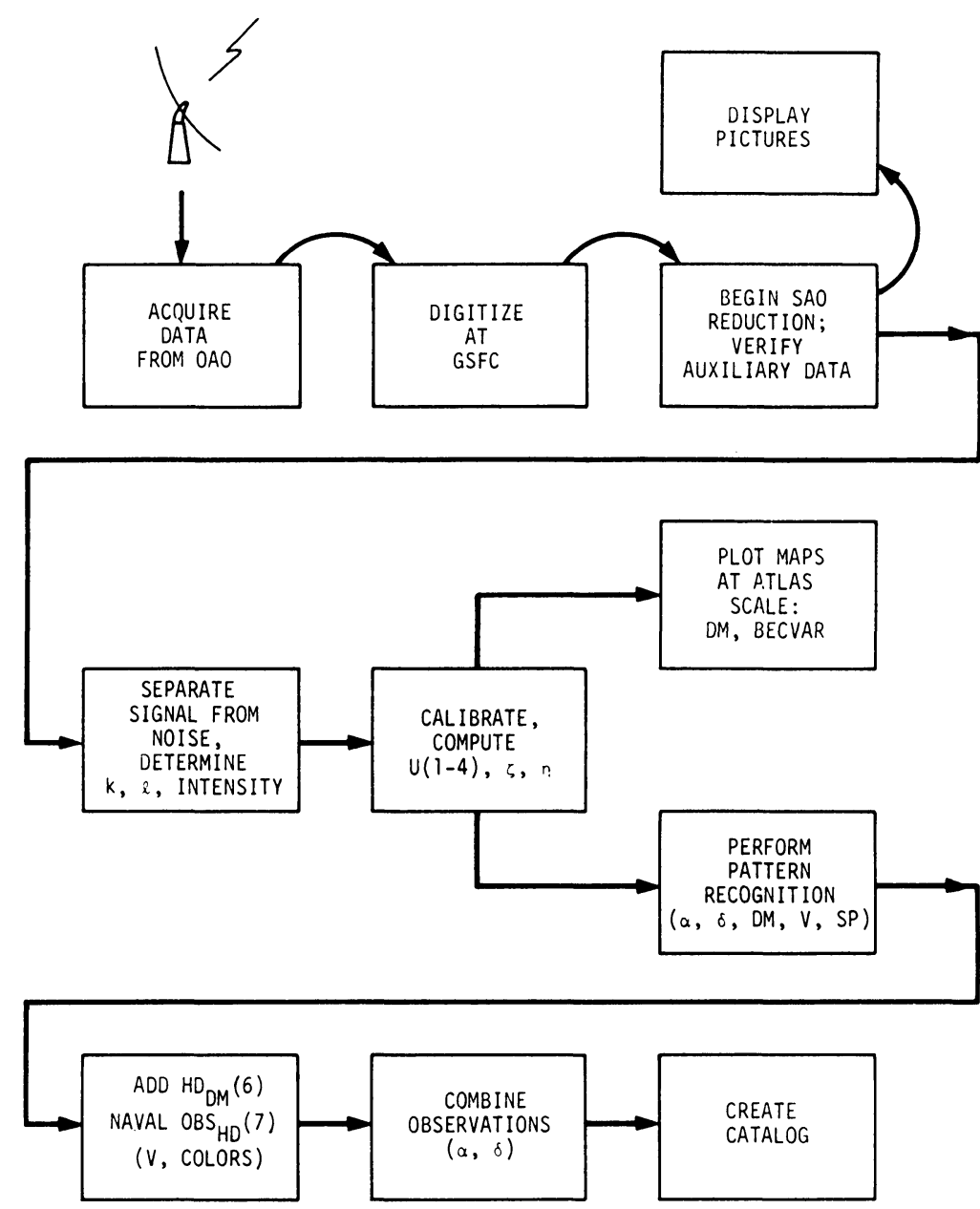

Fig. 5. The Celescope data reduction system.

in several ways to facilitate the inspection necessary for a uniform quality of output from the system. Our signal processing program produces a record for every object, including all its known parameters, its coordinates in the picture matrix $(k, l)$, and its observed intensity (I), which is defined as the sum of all non-noise contiguous elements making up the object, minus the background intensity for each such element.

After the computation of relative intensity, the data are reduced to ultraviolet magnitudes by using calibration data prepared before launch in laboratory tests and verified with orbital data taken throughout the satellite's lifetime. Principal features of the calibration system are an optimization procedure by the Fletcher-Powell method, used to refine the prelaunch calibration tables, and the utilization of repeated orbital observations of special sky areas to determine the time degradation of the system in orbit. The early calibration has been extensively discussed in Davis (1968); special 
techniques in current use are treated in Lundquist et al. (1971). The most recent determination of the standard error of unit weight, using only those observations accumulated before June of this year, gave the values reported in Table II.

Part of the prelaunch calibration was devoted to the conversion of picture matrix elements $(k, l)$ to a pseudoequatorial system $(\zeta, \eta)$ that, with knowledge of the satellite pointing $(\alpha, \delta)$ and of the moment of exposure, would be directly comparable to right ascension and declination. With the $\zeta, \eta$ coordinates, a small plot is made of each picture at the scale of the most useful atlases, the BD, $\mathrm{CD}$, and Bečvár. These plots are often useful in resolving difficult identifications. The $\zeta, \eta$ coordinates alone are capable of representing the $\alpha, \delta$ of an object within $\pm 10^{\prime}$.

\section{TABLE II}

RMS magnitude deviation for each camera

Camera $\quad$ RMS $\Delta m$

\begin{tabular}{ll}
1 & 0.17 \\
3 & 0.20 \\
4 & 0.19 \\
\hline
\end{tabular}

The $\zeta, \eta$ coordinates and the necessary information on pointing and moment of exposure are used in a pattern-recognition program that matches the observatione against a specially prepared list of early-type stars. Currently, by making composits 'pictures' from several actual pictures and matching stars in areas several degrees in diameter, we achieve satisfactory identifications for about $80 \%$ of our observations. The pattern-recognition program that we use was adapted from that employed for many years at SAO and based on the Christie-Dyson method of 'plate constants' (Arnold, 1970).

After satisfactory identifications are determined and checked for all our data, we add - for our own use and for the aid of others who will use the final catalog - magnitudes and colors in the UBV system, spectra, HD numbers, DM numbers, and magnitudes $\left(m_{v}, m_{p g}\right)$ if no UBV data are available. The major sources we utilized to accumulate these ground-based data, all in machine-accessible form, are as follows:

(1) The Celescope identification list, a compilation of several hundred references specially selected as containing data on the objects most likely to be seen by the instrument.

(2) The Henry Draper Catalogue (HD), obtained on 10 tapes from Yale University Observatory and reduced, for ease in handling, to a single tape and sorted by DM number.

(3) The Naval Observatory Catalogue of Blanco et al. (1968), which originally contained several observations for each entry and which we have reduced to a single entry per star. 
(4) The SAO Catalog, which we have also reduced to a single tape and sorted by DM number.

An important feature of our system is the ease with which we can rearrange all observations and catalog data so as to make the programs that operate on them efficient. For example, our initial processing through the calibration phase progresses one picture at a time; pattern recognition is performed with the data sorted into convenient blocks by pointing; ground-based data are added in order either by $D M$ number or by $H D$ number. Final combination of observations will be carried out with the data in order by right ascension and declination. The ease with which we can perform these rearrangements depends largely on our adherence to a uniform format for the magnetic tapes that are internal to our system.

\section{The Observations}

With these photometers, we have taken close to 9000 pictures, from about $50 \%$ of which we have currently reduced about 22000 observations of 5000 stars. The sky area covered during the exposures taken during our 16 months of operation is shown in Figure 6. Aside from a few pictures taken at high galactic latitude, we have concentrated principally on the galactic plane. As an illustration of the characteristics of our survey, I have compared our data on early-type stars with data from the HD (Cannon and Pickering, 1918-1924). Figure 7 compares the number of stars observed by Celescope and listed in the HD for spectral classes B and A. For these classes, it is apparent that the HD, with its 60 min exposures, is virtually complete to $m_{v}=8.3$ and that, for the areas our experiment observed with its $60 \mathrm{~s}$ exposures, Celescope has a faint limit around $m_{v}=8.1$. Only stars with known spectral class have been included in this diagram; a considerable number of faint stars in our data are of unknown spectral classes.

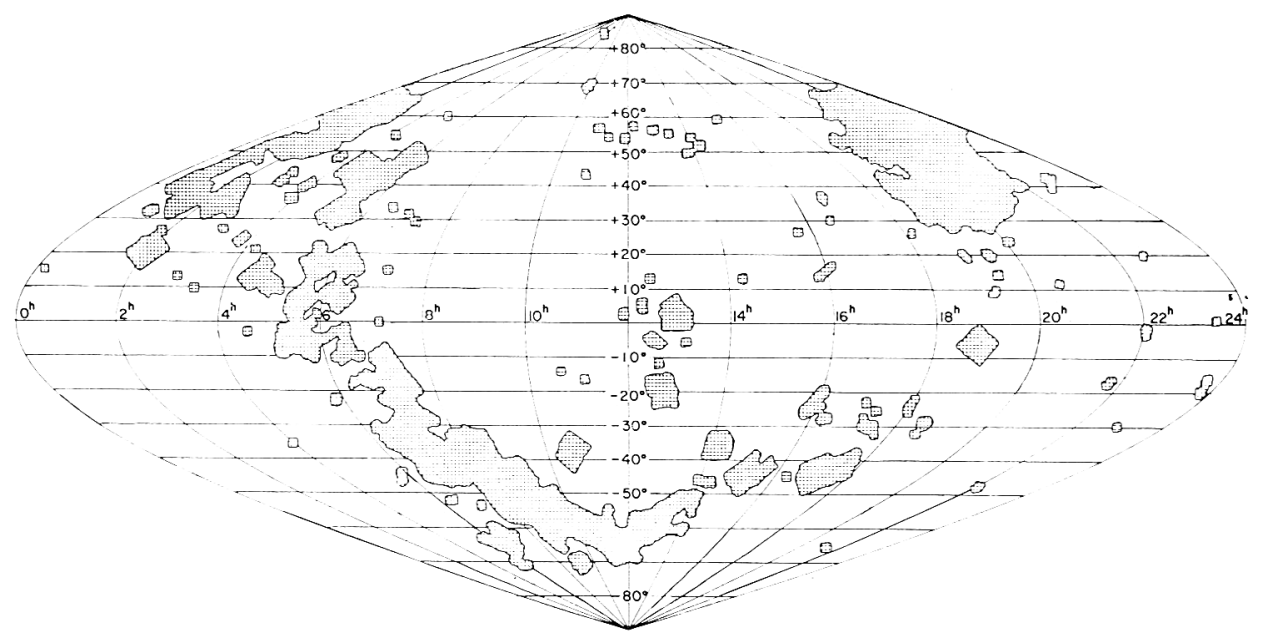

Fig. 6. Sky areas observed by Celescope, by $(\alpha, \delta)(1950)$. 


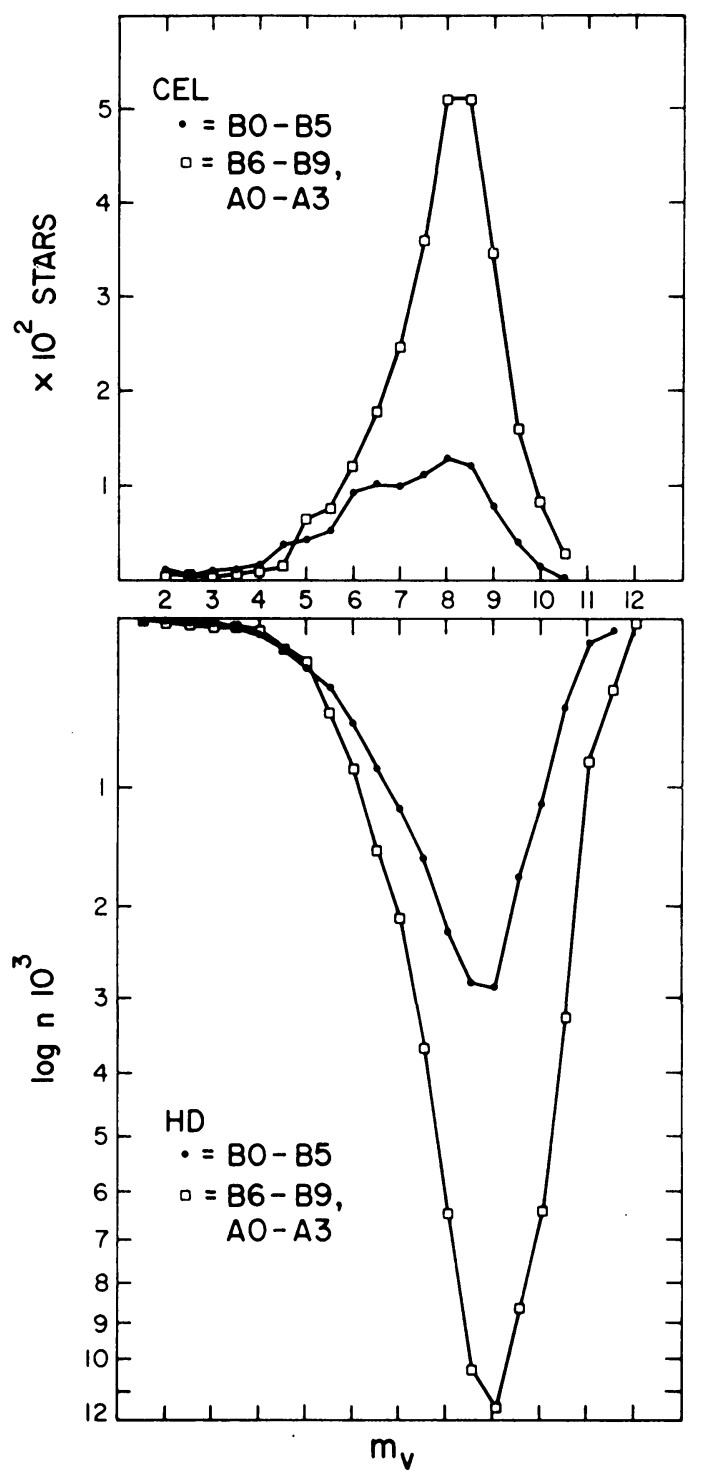

Fig. 7. A comparison of the number of $B$ and $A$ stars observed by Celescope and listed in the HD: $\mathrm{O}=\mathrm{B} 0-\mathrm{B} 5 ; \square=\mathrm{B} 6-\mathrm{B} 9, \mathrm{~A} 0-\mathrm{A} 3$.

A comparison of the $\mathrm{O}$ and Wolf-Rayet stars seen by Celescope and recorded in the HD is shown in the pair of diagrams in Figure 8. The two diagrams are drawn in a three-dimensional Cartesian system, with axes corresponding to $(\alpha, \delta)_{1950}$ and with the number of stars in each sky area bounded by the limits represented. The Great Rift and the Magellanic Clouds are prominent. Dashed lines on the HD part of the diagram indicate an area not observed by Celescope. It may be of interest to note that 

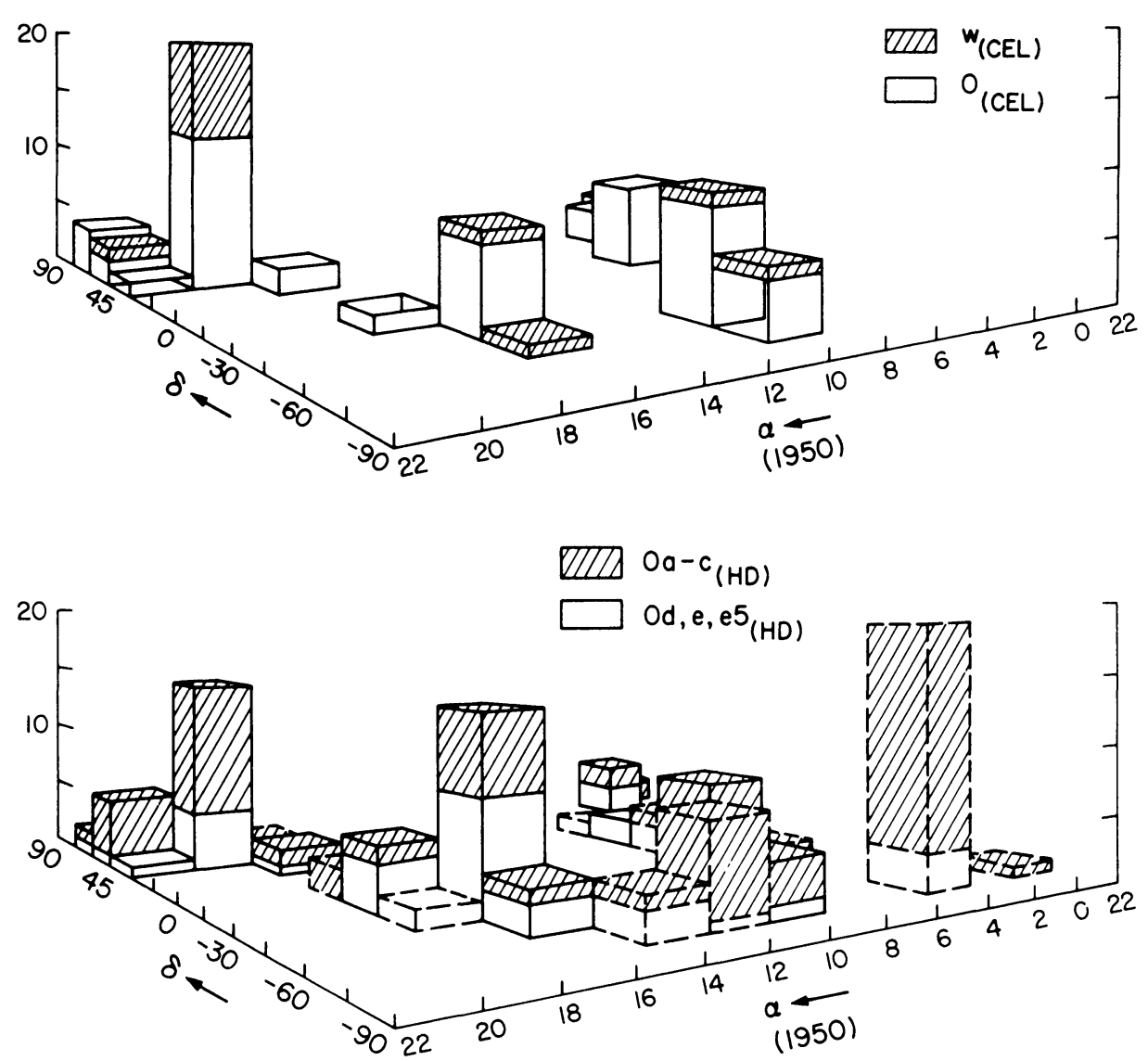

Fig. 8. The $\mathrm{O}$ and Wolf-Rayet stars observed by Celescope (above) and listed in the HD (below). Right ascension, declination, and number of stars are shown.

Shapley and Cannon (1924) in their discussion of the data from the HD did not give information concerning the $\mathrm{O}$ stars, which $\mathrm{I}$ furnish here, and that their numerical results for B and A stars differ from mine by several percent (see Table III). Both tallies were, of course, performed on the same catalog, theirs for 220570 stars while the catalog was in manuscript and mine by computer on 225300 stars in the published HD.

\section{TABLE III}

Early-type HD stars tabulated by Shapley and Cannon (1924) and in the present paper

Spectral class Shapley and Cannon This paper $\Delta \%$
O

$-$

168

B0-B5

3567

3230

9
$\mathrm{B} 8, \mathrm{~B} 9, \mathrm{~A} 0-\mathrm{A} 3$

64259

62477 


\section{Documentation of Data and Methods}

My attention has been brought many times to the difficult task of adequate documentation of the data and results of the experiment. Figure 9 illustrates the amounts of material we have accumulated, first in the laboratory and later in orbit. The largest are the data themselves; the supporting documents are more manageable, perhaps $10 \%$ of the original; and the summary documents contain only the vital information and reduce the sheer weight of paper to a fraction of $1 \%$ of the original. It can be extremely difficult to document adequately an enterprise of this size, but we have found that certain principles can be profitably followed:

(1) Any document or related group of documents of more than 200 to 300 pages

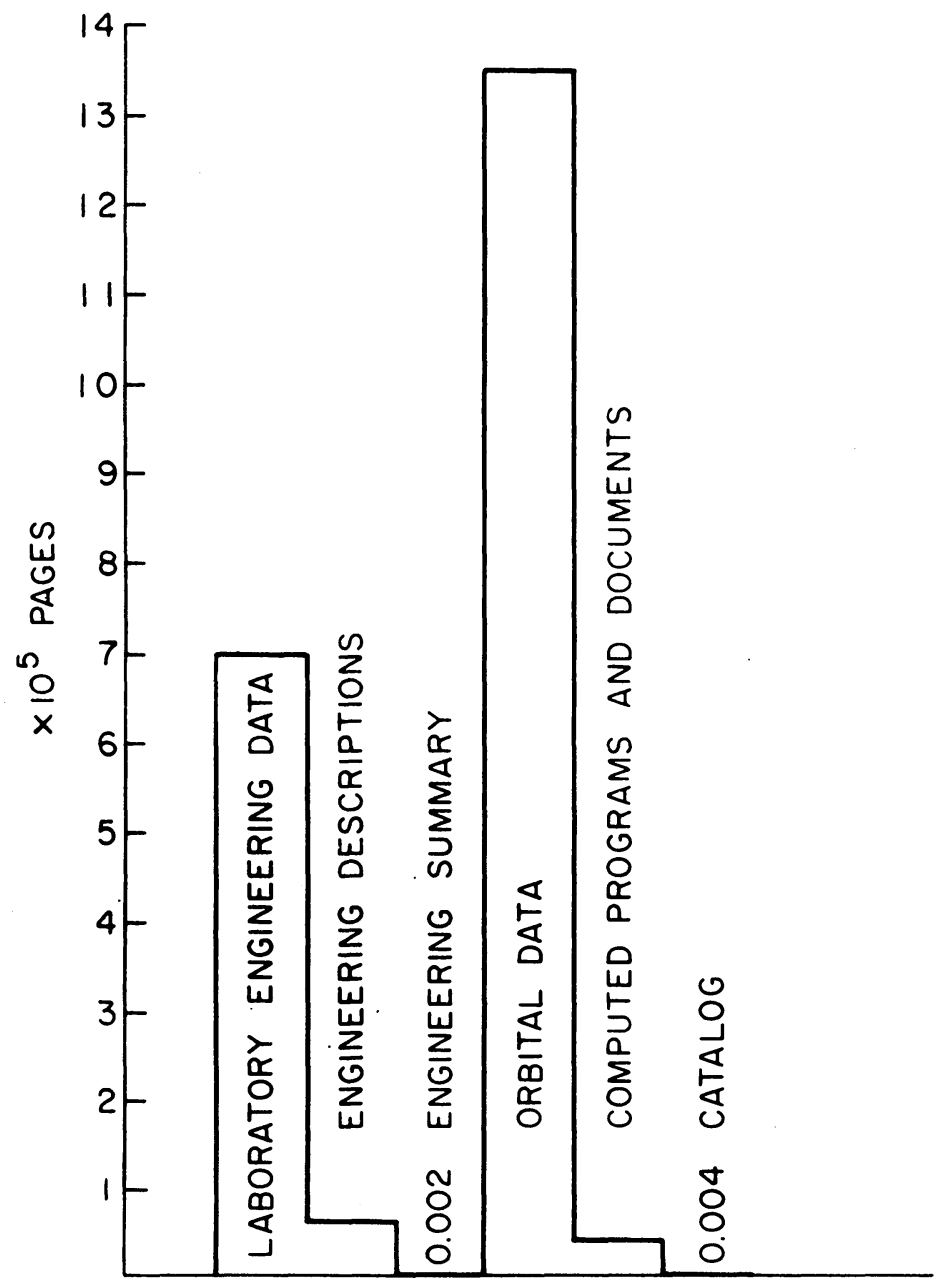

Fig. 9. Celescope documentation. 
needs a summary not longer than a page or so. Failure to provide such a summary may result in misplacement or loss of important information.

(2) Documents of the same kind should be handled together in the documentation; engineering documents requiring special treatment are not always needed for data analysis, and vice versa. It can be useful to have an individual knowledgeable in a special field in charge of the documentation for his type of information. His documentation should conform to certain standards and dovetail with the complementary documentation from the other parts of the project.

(3) Data (in the digitized form in which we use it) and computer programs, if supplied with consistent and appropriate headings and comments, are often their own best documentation. However, a computer program without the name of its author, dates of creation and revisions, and a multitude of explanatory comments placed within the listing is virtually useless to anyone else who may need to use or revise it.

(4) In the description of the essentials of a catalog or compendium of data such as ours of ultraviolet observations, it is important to the user that the compiler specify as completely as possible what was gone into the catalog:

(a) First, a well-organized description of the purpose, design, and preparation of the list should be given.

(b) Second, the compiler should try to remember that what is familiar to him is not necessarily familiar to those who will use his work.

(c) Third, it would be extraordinarily useful if original data presented for the first time in a list could be distinguished from the data merely copied from somewhere else. In this age of lists often taken directly from computer printout or CRT, no easy substitute for italics may be available, but column headings or perhaps single and double vertical lines scribed on the page might be effectively utilized.

(d) Finally, if the introduction to the list is necessarily lengthy and complex, an index would be a useful addition.

Two excellent examples of list specification come to mind: the Henry Draper Catalogue, where all the spectral classes are explicitly defined and, where room did not allow greater discussion of special methods, a specific reference is given; and the N30 (Morgan, 1950), where Morgan discussed not only the entire reasoning behind his catalog but also his whole system of reduction, in a completely clear and understandable fashion. Some catalogs do not come up to these standards because they give either so little information that their scheme is insufficiently explained or so much that it actually impedes use of the catalog. The user must in effect analyze the catalog before he can use it!

In this light, I have one last figure that illustrates a problem well known to those working with stars and their identifications. In Figure 10 I have plotted, for every $1^{\circ}$ of declination, the number of stars in each of the four Durchmusterungen. When the HD was constructed, Pickering and Cannon decided that south of the last complete zone of the SBD they would use the CD, and from $-52^{\circ}$ to the south pole, the CPD for their identifications. If they based their choice on the number of stars available from the two southern-hemisphere catalogs, then their choice was very wise. 


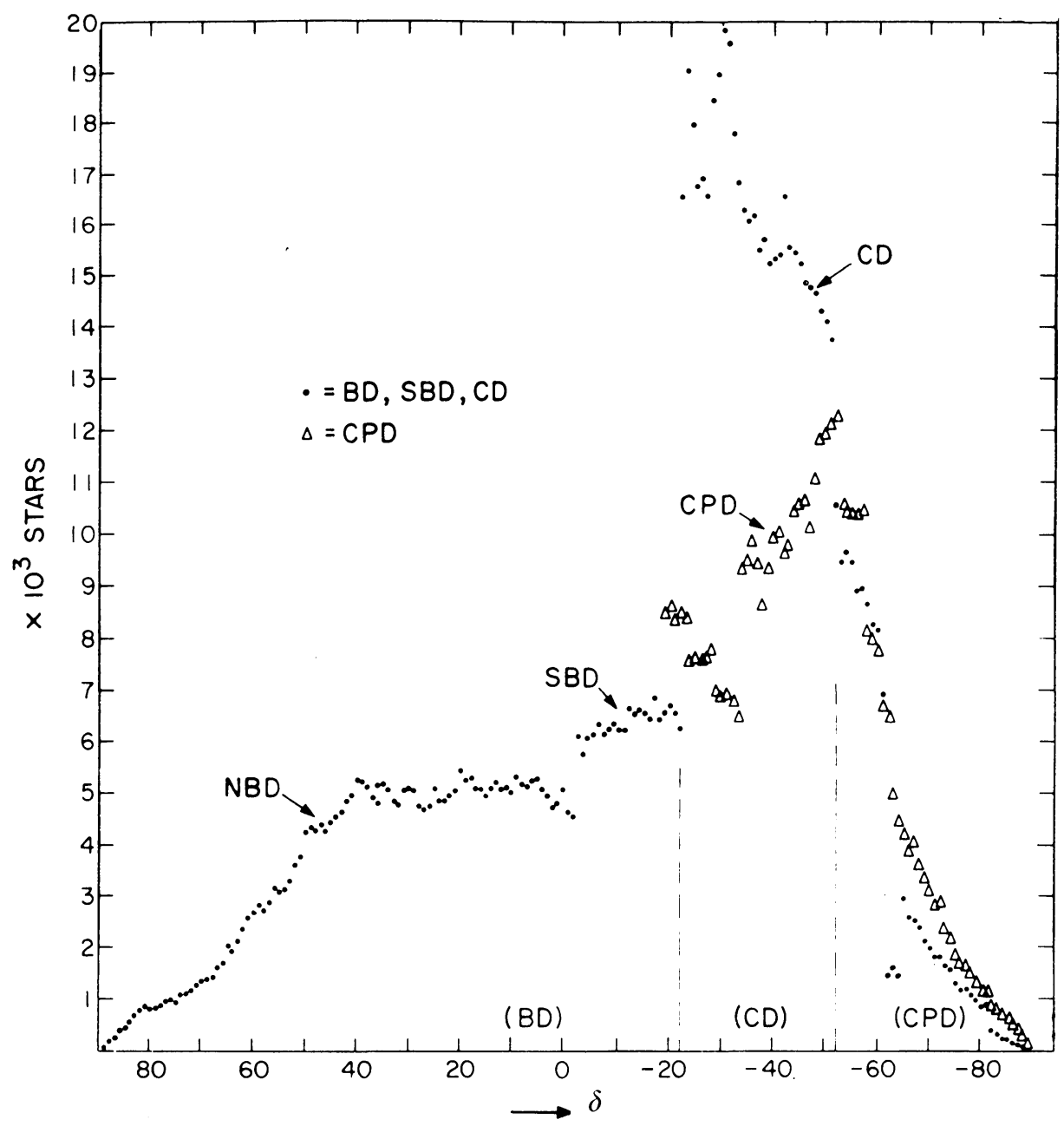

Fig. 10. The number of stars in each Durchmusterung, by declination; HD convention indicated in parentheses.

They did not, however, use the $\mathrm{CD}$ in the zones where the $\mathrm{BD}$ overlapped with it. The existence of the two DM in the south, however, has caused some difficulty, and I should like to hope that future list and catalog compilers adhere to a single convention in this regard. Three choices suggest themselves:

(1) Always use the CD.

(2) Always give both CD and CPD numbers.

(3) Adopt the Henry Draper convention, using CD numbers north of $-52^{\circ}$ and CPD numbers south of it.

My personal preference is toward the HD convention (even to the exclusion of the somewhat more numerous $\mathrm{CD}$ stars between $-19^{\circ}$ and $-22^{\circ}$ ), but I vigorously recom- 
mend that, at the least, in any list a DM number, where it exists, should always accompany data reported for an object.

\section{Acknowledgements}

I am indebted to Peter Collins for his assistance in preparing some of the data for this paper, and to C. Payne-Gaposchkin for helpful discussions. This work was supported in part by contract NASA-1535 from the National Aeronautics and Space Administration.

\section{References}

Arnold, D.: 1970, Photoreduction Manual, internal SAO document.

Blanco, V. M., Demers, S., Douglass, G. G., and Fitzgerald, M. P.: 1968, 'Photoelectric Catalogue: Magnitudes and Colors of Stars in the $U, B, V$, and $U_{c}, B, V$ Systems', Publ. Naval Obs., 2nd Ser. XXI.

Cannon, A. J. and Pickering, E. C.: 1918-1924, 'The Henry Draper Catalogue', Ann. Astron. Obs. Harvard Coll. 91-99.

Celescope Staff 1971, 'Performance Evaluation of the Celescope Experiment', Rpt. to NASA, July. Davis, R. J.: 1968, Smithsonian Astrophys. Obs. Spec. Rpt., No. 282.

Lundquist, C. A., Deutschman, W. A., and Eng Young, R.: 1971, 'Digital Processing Techniques Used in Determining Stellar Ultraviolet Magnitudes from OAO-Celescope Image Data', presented at the Symposium on the Processing of Telescopic Images.

Morgan, H. R.: 1950, 'Catalog of 5,268 Standard Stars, 1950.0. Based on the Normal System N30', Astronomical Papers of the American Ephemeris and Nautical Almanac XIII.

Nozawa, Y.: 1969, in T. D. McGee, D. McMullan, and E. Kahan (eds.), Advances in Electronics and Electron Physics 28B, Academic Press, New York, p. 891.

Shapley, H and Cannon, A. J.: 1924, Harvard Reprint No. 6. 